\title{
LARES: A New Satellite Specifically Designed for Testing General Relativity
}

\author{
Antonio Paolozzi, ${ }^{1,2,3}$ Ignazio Ciufolini, ${ }^{3,4}$ Claudio Paris, ${ }^{2,3}$ and Giampiero Sindoni ${ }^{2}$ \\ ${ }^{1}$ Scuola di Ingegneria Aerospaziale, Sapienza Università di Roma, Via Salaria 851, 00138 Roma, Italy \\ ${ }^{2}$ Dipartimento di Ingegneria Astronautica Elettrica ed Energetica, Sapienza Università di Roma, Via Eudossiana 18, 00184 Roma, Italy \\ ${ }^{3}$ Centro Fermi, Via Panisperna 89, 00184 Roma, Italy \\ ${ }^{4}$ Dipartimento di Ingegneria dell'Innovazione, Università del Salento, Via per Monteroni, 73100 Lecce, Italy
}

Correspondence should be addressed to Antonio Paolozzi; antonio.paolozzi@uniromal.it

Received 30 August 2014; Accepted 24 November 2014

Academic Editor: Linda L. Vahala

Copyright (C) 2015 Antonio Paolozzi et al. This is an open access article distributed under the Creative Commons Attribution License, which permits unrestricted use, distribution, and reproduction in any medium, provided the original work is properly cited.

It is estimated that today several hundred operational satellites are orbiting Earth while many more either have already reentered the atmosphere or are no longer operational. On the 13th of February 2012 one more satellite of the Italian Space Agency has been successfully launched. The main difference with respect to all other satellites is its extremely high density that makes LARES not only the densest satellite but also the densest known orbiting object in the solar system. That implies that the nongravitational perturbations on its surface will have the smallest effects on its orbit. Those design characteristics are required to perform an accurate test of frame dragging and specifically a test of Lense-Thirring effect, predicted by General Relativity. LARES satellite, although passive, with 92 laser retroreflectors on its surface, was a real engineering challenge in terms of both manufacturing and testing. Data acquisition and processing are in progress. The paper will describe the scientific objectives, the status of the experiment, the special feature of the satellite and separation system including some manufacturing issues, and the special tests performed on its retroreflectors.

\section{Introduction}

Typical satellite lifetime is about 10 years, but for passive satellites such as LARES (LAser RElativity Satellite) there is no such estimate because duration is basically only limited by the stability of the optical properties of the retroreflectors and therefore is extremely long; for example, the LAGEOS satellite is efficiently operating since 1976, that is, since 38 years. Passive satellites often carry Cube Corner Reflectors (CCRs) that have the property of reflecting back to the laser ground stations a laser pulse regardless of the CCR orientation. By measuring the exact flight time of the laser pulse it is possible to measure the distance with errors that can be as low as few millimeters. Even the lunar CCRs are operational since year 1969 [1] when the Apollo 11 mission left an array of CCRs on the Moon. However a severe degradation on the reflecting performances of all lunar CCRs arrays has been observed [2]. The causes are not still well identified but seem related to the specific lunar environment such as particle deposition on CCR front face due, for instance, to micrometeorites induced dust. Based on that information it can be hypothesized that a lower limit for LARES lifetime could be about 50 years. Besides that, LARES, as the densest orbiting body in the solar system, will reenter very slowly. Orbital lifetime for LARES can be estimated of several thousand years [3] which is a remarkable duration considering the relatively low orbit. According to other estimates the reentry will happen in about 100 thousand years. The possibility to rely on a long mission duration is an interesting point, because LARES experiment requires both the accurate orbit determination and the most accurate gravitational and nongravitational perturbation estimation. Measurement techniques and knowledge of gravitational perturbations have been dramatically improving over time in the last decades. It is expected that in the future further improvements will be achieved so that a longer lifetime for 
LARES could allow a more accurate experiment. This trend has been already experienced with two other passive satellites: the LAGEOS satellites. In [4] a first observation of the LenseThirring effect (a General Relativity prediction) has been obtained using EGM-96 gravitational field released in 1996. Later [5] a more accurate measurement has been obtained with an accuracy of about $10 \%$ [6] thanks to the GRACE space mission and to the accurate release of the EIGENGRACE02S gravitational field [7].

\section{Scientific Objectives}

Although LARES data can be exploited by geodesists to improve the International Terrestrial Reference Frame (ITRF) by about 25\% [8] and to perform other geodesy and geodynamic studies, its main objective is to test the gravitational theory of General Relativity. It has been confirmed by all the tests performed so far starting from the famous Mercury perigee shift [9] to the light deflection due to a mass [10], to the equivalence principle $[11,12]$, to the time dilation in the presence of a mass (Gravity Probe A experiment) $[13,14]$, and to the time-delay in the propagation of electromagnetic waves near a mass. Also gravitational waves, although not directly observed by the many detectors developed so far, have been indirectly observed through their effects on binary pulsars [15]. Frame dragging is another intriguing effect guessed by Einstein before the publication of the General Relativity theory [16]: a current of mass-energy will drag spacetime, time [17], and the local inertial reference frames. The axis of an inertial reference frame orbiting Earth, a gyroscope, will not always point towards "fixed" stars, as in GalileiNewton mechanics, because it will be dragged by the Earth rotation. The effect is very small but measurable: in polar orbit at $650 \mathrm{~km}$ it would amount to only $39.2 \mathrm{milliarcsec} /$ year. The most direct and intuitive way to physically realize an inertial reference frame is through three mutually orthogonal gyroscopes in free fall. This type of reference frame has been actually manufactured and launched in 2004 in a polar orbit at $650 \mathrm{~km}$ altitude after 40 years of development and has been called the Gravity Probe B experiment. It has been among the most expensive experiments ever built because of its very high technological achievements. Some unpredictable effects on the four spherical gyroscopes limited the accuracy in the measurement of the drift of the gyroscope axes due to frame dragging to about $19 \%$ [18]. In principle, another way of realizing a gyroscope is to consider the vector orthogonal to the orbital plane of a satellite in the ideal case of an unperturbed orbit and of a perfectly spherical gravitational field, that is, for a motion under a central force. Frame dragging will cause the rotation of the nodal line of the satellite. This rotation is called Lense-Thirring effect and was derived from General Relativity [19]. It amounts to 118 milliarcsec/year for LARES. As will be described more in detail later, LARES orbit approaches better than any other artificial body, a geodesic in spacetime [20], and therefore is so far the best realization of a test particle, thus minimizing nongravitational perturbations.

Concerning the nonspherical Earth gravity field, its effect on the orbit has to be determined very accurately.
Mathematically the gravity field of a planet can be expanded in spherical harmonics [21]. The ones critical for LARES experiment are the so-called even zonal harmonics: $J_{2} J_{4} \cdots J_{2 n}$. The effect of $J_{2}$ (which physically corresponds to the Earth polar flattening) on the LARES orbit is similar to frame dragging but million times bigger. That means that the knowledge of the Earth gravitational field has to be very accurate to be able to distinguish the Lense-Thirring effect from the effect of $J_{2}$ and of the other even zonal harmonics. Unfortunately the present accuracy of gravitational fields is not sufficient by itself to meet the objective. In fact even the most accurate gravitational fields determinations available today, such as EIGENGRACE02S, have uncertainties on the effect of $J_{2}$ that are still of the order of the Lense-Thirring effect. A way out of this problem has been proposed by combining the results of the two LAGEOS satellites. This way it is in fact possible to eliminate the effect of $J_{2}$ and consequently of its uncertainty, reaching a final accuracy of the Lense-Thirring effect of about 10\%. The LAGEOS 1 and 2 satellites were launched in 1976 by NASA and in 1992 by NASA and ASI, respectively. The $10 \%$ error is mainly due to the uncertainties of $J_{4}$ that will be eliminated with the new LARES satellite as shown by the error analysis performed in [22] and, using an indepented approach, in [23]. Since the 17th of February 2012, when the first laser return signal was received from the orbiting LARES satellite, millions of ranging data have been acquired by ILRS [24]. However there are some other periodical perturbations related to the Luni-Solar tides whose uncertainties can be eliminated by averaging and fitting for their orbital effects over a sufficiently long period of time. That means it will take a few more years before one can further reduce the error in the Lense-Thirring effect measurement from the $10 \%$, already obtained, to about $1 \%$. On the other hand from the first data analysis it has been verified that LARES is indeed the best test particle today available in the solar system [25].

\section{Mission Description}

The opportunity provided by the European Space Agency (ESA) for the qualification launch of VEGA was taken by the Italian Space Agency (ASI) that decided to support LARES mission in 2008. This launch opportunity was also taken by three more Italian spacecraft (ALMASat-1 from University of Bologna and two Cubesats from Politecnico of Turin (EST@R) and Sapienza University, Gauss (UniCubeSat-GG)). Besides those three satellites, the educational program of ESA allowed the launch of five more Cubesats from other European universities. In Figure 1 the LARES system is reported. In the center LARES satellite mounted on the separation system (SSEP) is shown which is fixed on the support system (SSUP). On the top right is ALMASat-1 and on the left and on the right one and two PPODs, respectively. A PPOD is a standard interface specifically designed to contain and release up to three Cubesats. Due to the high number of payloads released on the same orbit and the need to fulfill the space debris mitigation measures, an assessment of collision risk was performed to demonstrate that such a risk was negligible. 


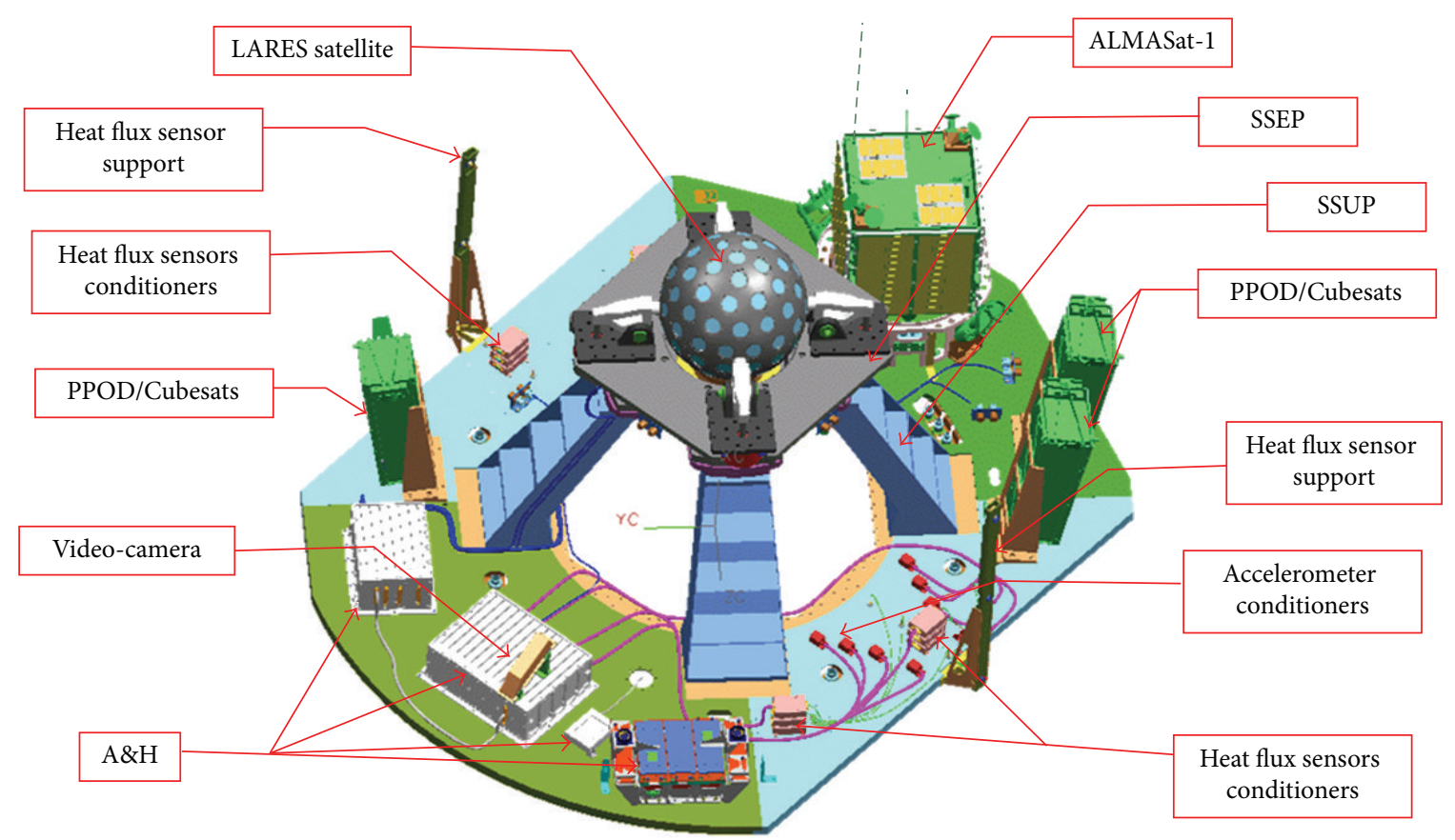

FIGURE 1: LARES system.

On the 13th of February 2012, VEGA lifted off the ESA spaceport in French Guyana. LARES, the first satellite to be separated after 55 minutes, was successfully inserted into a circular orbit at $1450 \mathrm{~km}$ altitude and 69.5 degrees inclination $[26,27]$. After that, the last stage ignited for the third time going to an elliptical orbit with the perigee lowered at about $350 \mathrm{~km}$. After about 15 minutes the seven Cubesats were separated first, followed by ALMASat-1. The mission was a success. VEGA demonstrated high accuracy and all the satellites were separated exactly in time. An on board camera recorded all the phases of the flight including the separations. To provide a quantitative estimate of the orbital injection accuracy, in Table 1 the nominal and the measured orbital parameters of LARES are reported.

\section{Satellite and Separation System: Design and Manufacturing}

The satellite requirements could be summarized in few words: LARES should be an "almost perfect test particle." In fact theoretically an ideal test particle trajectory will be unaffected by perturbations such as solar radiation pressure and atmospheric drag thus allowing to probe the gravitational field very accurately. An engineering realization of a test particle is a very dense and passive satellite, while another relies on an active control of satellites. These are called dragfree satellites: the test particle is inside and is not subjected to the abovementioned perturbations because of protection by the spacecraft exterior. Noncontact sensors will measure the differential motion between the test particle and the satellite. Small rockets on the satellite compensate the perturbation forces acting on it so that the test particle never touches
TABLE 1: LARES orbital parameter.

\begin{tabular}{lcc}
\hline Orbital parameters & Nominal & Measured (min-max) \\
\hline Inclination (degrees) & 69.5 & $69.45-69.56$ \\
Eccentricity & 0 & $0-0.002$ \\
Altitude $(\mathrm{km})$ & 1450 & $1430-1470$ \\
\hline
\end{tabular}

the satellite internal surfaces and moves as a drag-free body. Which one of those two approaches is the best is difficult to tell. They are not always interchangeable. For instance, the four gyroscopes of GPB required necessarily a drag-free spacecraft while for an engineering realization of an orbiting test particle both approaches could be feasible: a drag-free test mass and a very high mass-to-surface ratio satellite. The level at which drag-free is guaranteed is measured in $\mathrm{pm} / \mathrm{s}^{2}$ which is 0.4 and 40 for LARES and GPB, respectively. Of course on LARES, due to its simple shape, it is easier to estimate the effects of the perturbations that can be therefore taken into account in the satellite motion ultimately leading with such a small value of residual acceleration.

Given the abovementioned requirements, the design of LARES has undergone several changes before the critical design review. The first idea was to look at similar satellites such as LAGEOS. The main body of LAGEOS was constituted by three parts bolted together with a copper beryllium tension stud and two nuts (Figure 2).

The central cylindrical part was made of a copper alloy with a density of about $8500 \mathrm{~kg} / \mathrm{m}^{3}$ while the two external shells were made of Al 6061 with a density of $2700 \mathrm{~kg} / \mathrm{m}^{3}$. This choice was reasonable being a good compromise between the requirement to realize a dense satellite, which better 
TABLE 2: Characteristics of laser ranged satellites. ${ }^{*}(M / S)_{\text {Satellite }} /(M / S)_{\text {LAGEOS }}$, that is, the values of mass-to-surface ratio, are normalized with respect to that of LAGEOS.

\begin{tabular}{|c|c|c|c|c|c|}
\hline & LARES & LAGEOS & LAGEOS 2 & STELLA & STARLETTE \\
\hline Mass (kg) & 386.8 & 407 & 405 & 48 & 47 \\
\hline Radius (mm) & 182 & 300 & 300 & 120 & 120 \\
\hline Number of CCRs & 92 & 426 & 426 & 60 & 60 \\
\hline Normalized $M / S$ Mass/surface* & 2.6 & 1 & 0.99 & 0.72 & 0.73 \\
\hline CCRs area/total area & $26 \%$ & $43 \%$ & $43 \%$ & $28 \%$ & $28 \%$ \\
\hline Unmodeled residual accel. $\left(\mathrm{pm} / \mathrm{s}^{2}\right)$ & 0.4 & $1-2$ & $1-2$ & 40 & 40 \\
\hline
\end{tabular}

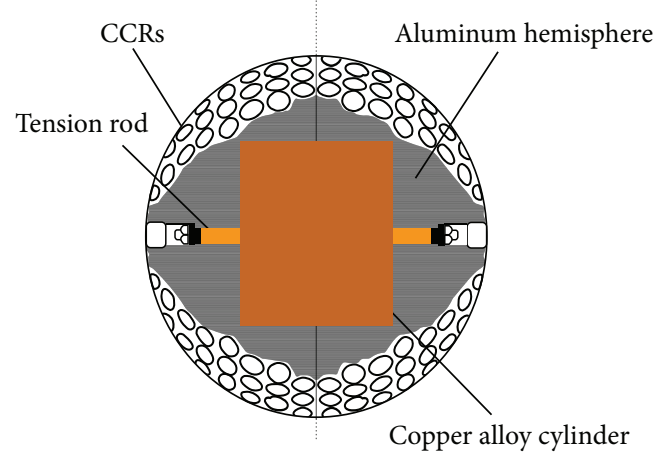

FIGURE 2: LAGEOS satellite main components.

approximate a test particle, and the manufacturing needs. The most demanding manufacturing operations have to be performed on the surface, and aluminum alloy is relatively easy to machine and its behavior in space is very well known. Starting from this type of design a first improvement for LARES was to consider, for the internal part, a higher density material. This solution, for instance, has been adopted for two laser ranged satellites: Stella and Starlette. In those two satellites the internal core was made of depleted uranium. Safety issues and the low popularity of this material prevented their use for LARES. Another material with almost the same density as depleted uranium is tungsten. Other materials with higher density such as iridium and osmium were not considered mainly for their high cost. Finally the best solution for the construction of the main body of the satellite was instead the most obvious although not the easiest one: to machine the satellite out of one single piece of tungsten alloy [28]. This way it is possible to obtain a very high density satellite. Furthermore building the satellite in one single piece reduced also thermal thrust perturbation which is caused by the anisotropic photon emission due to temperature gradients on the surface of the satellite. Temperature distribution of objects in vacuum is in fact dominated by thermal contact conductance; that is, it is a function of the number of components bolted or clamped together. With this design the satellite body contribution to thermal thrust was therefore minimized. Thermal thrust contribution of each single CCR is instead basically unmodified with respect to the LAGEOS design. Some alternative solutions to reduce thermal thrust from CCRs were studied in [29]. Considering that any strategy, aimed to reduce the contribution to thermal thrust of each CCR, would have affected sensibly the optical behavior of the CCR and, considering the short amount of time to launch, it was decided for a more conservative design of the CCRs and relevant mounting system. Anyway the CCR-to-metal surface ratio is lower for LARES with respect to LAGEOS [30] by a factor of 1.65 (Table 2); therefore the global optical design of LARES resulted in a lower contribution of CCR and relevant mounting systems to LARES thermal thrust.

Indeed with this single body design, entirely made of tungsten alloy, there were some counter indications: first it is almost impossible to obtain a sphere without defects using conventional techniques such as casting; second it was not guaranteed to have good tolerances on a material never used for space constructions. Concerning the first point, the liquid phase sintering process [31], used by the tungsten provider, is exempt from defects. Tungsten particles (95\% weight) are embedded in a matrix of $\mathrm{Cu}-\mathrm{Ni}(5 \%)$. Furthermore the center of mass of the semifinished sphere was practically coincident with its geometric center. In Figure 3 a micrograph clearly showing the two phases is reported. Indeed using Energy Dispersive Spectroscopy (EDS) analysis it could be recognized that the particles are made of pure tungsten, while the matrix around them is constituted by $58 \% \mathrm{Ni}, 13 \% \mathrm{Cu}$, and $29 \% \mathrm{~W}$ in weight.

Concerning the second point, the use of particular manufacturing parameters, the frequent change of machining tools, and a proper machining test campaign allowed reaching tolerances equal to or better than what was obtained on aluminum alloy laser ranged satellites. A particular problem arose with the CCR mounting system shown in Figure 4. It was difficult to manufacture the tungsten alloy screws due to the granular structure shown in Figure 3. In fact machining 


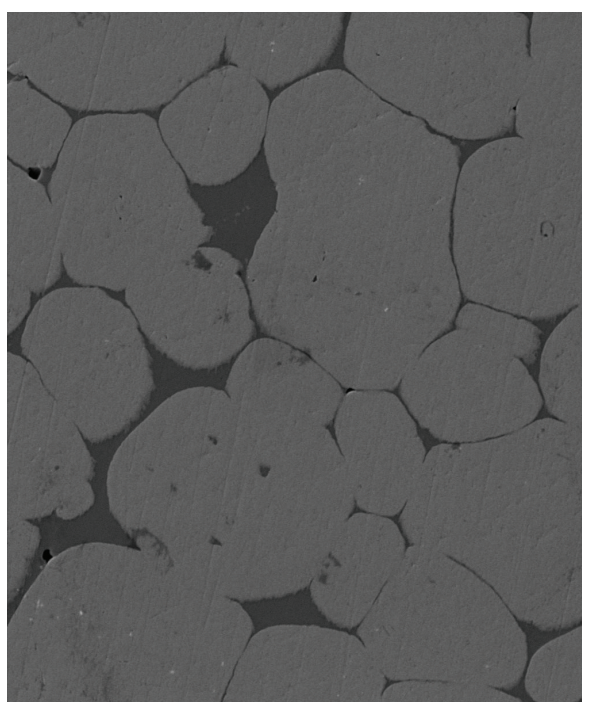

FIGURE 3: Micrograph of the tungsten alloy used for LARES. Average grain dimension $50 \mu \mathrm{m}$.

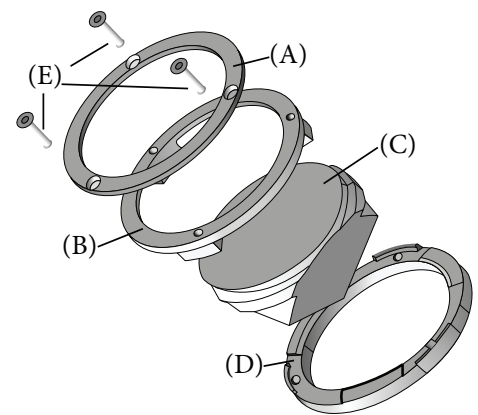

(a)

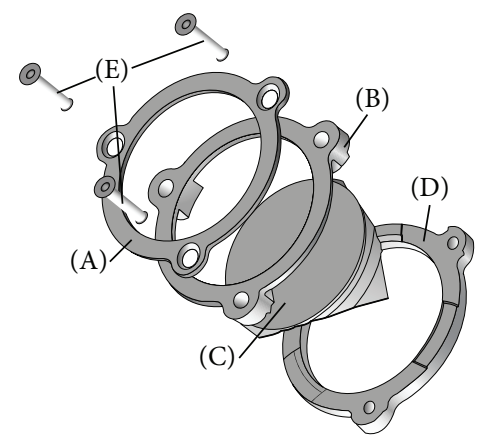

(b)

FIGURE 4: Comparison of LAGEOS (a) and LARES (b) cube corner reflector mounting systems. (A) Retainer ring, (B) plastic upper mounting ring, (C) CCR, (D) plastic lower mounting ring, and (E) screws.

introduced a high concentration of defects so it was prepared, in substitution, a procedure for plastic forming (rolling). The diameter of the screws was increased from two millimeters of the analogous aluminum LAGEOS CCR screws to three millimeters of tungsten alloy screws. As a consequence of this increase, the aluminum retainer ring shape was changed as

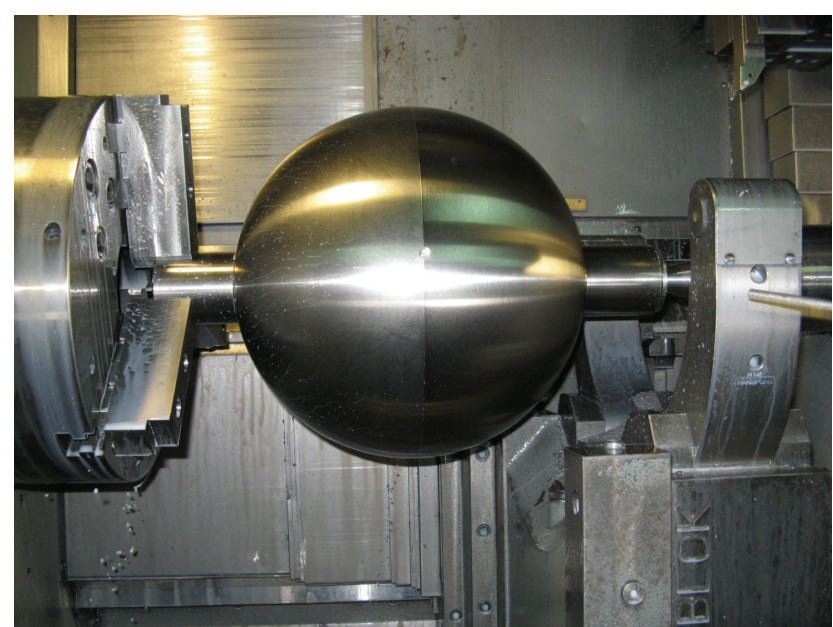

FIgURE 5: Semifinished sphere mounted on the lathe machine.

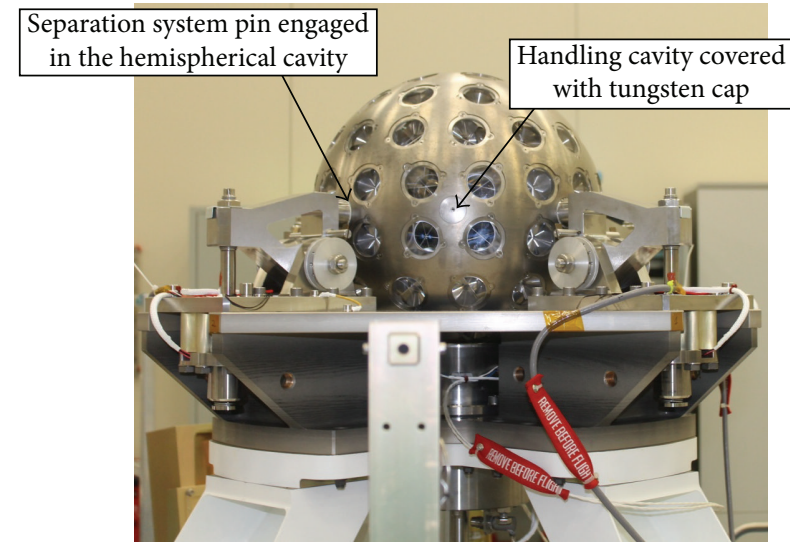

FIGURE 6: LARES satellite mounted on the separation system.

shown in Figure 4. Also the upper and lower plastic mounting rings were redesigned.

The machining of the $92 \mathrm{CCR}$ cavities plus eight more equatorial cavities, used for handling and interfacing the separation system, started from the semifinished sphere reported in Figure 5. The presence of the two protruding cylinders required to clamp it during the first phases of machining can be noticed. In this figure one can see the first operation on the lathe machine required to bring the final radius from 186 to the nominal value of 182 millimeters.

On the equator of the satellite there are four handling cavities that have been closed with tungsten alloy caps after final assembling with the separation system (Figure 6).

Also four hemispherical cavities located at $45^{\circ}$ from the previous ones have been designed and manufactured with a specifically designed tool. Those cavities could not be covered afterward in orbit separation and therefore to limit the uncertainty of their contribution to atmospheric drag their size was minimized. This was not a trivial task because the satellite was maintained in place just with the pressure of the four brackets of the separation system engaged in the hemispherical cavities. There were no clamps or bolts but 


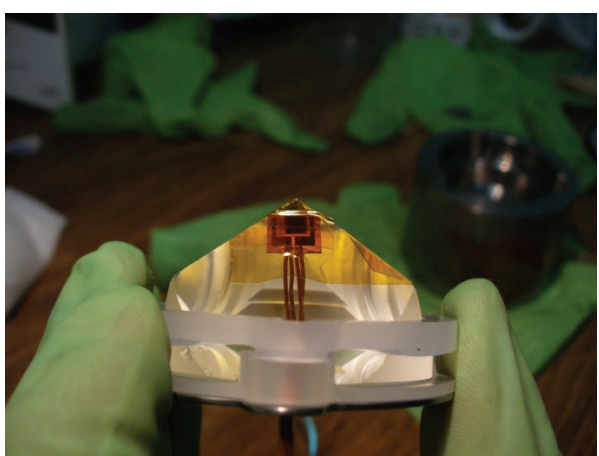

(a)

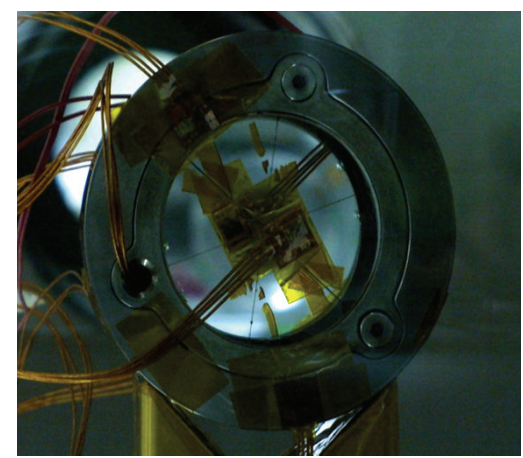

(b)

FIGURE 7: LARES cube corner reflector. PT100 glued close to the apex of CCR (a) and on the front face (b).

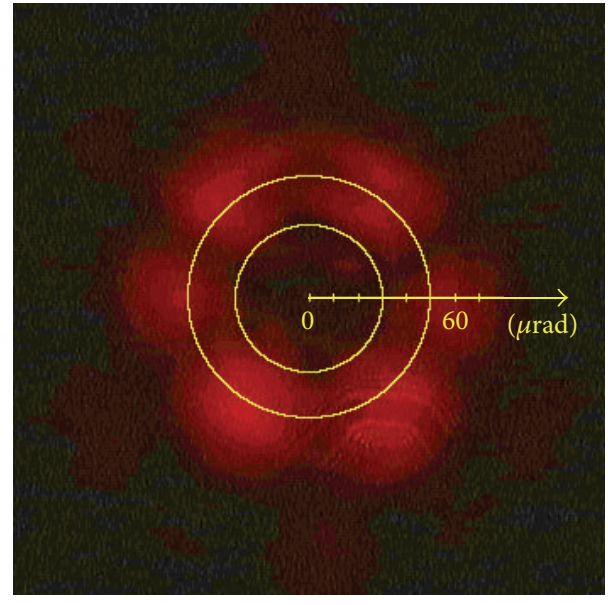

FIGURE 8: FFDP, in air, of CCR n.51 at room temperature. The dihedral angles offset from $90^{\circ}$ are for this CCR 1.14, 1.43, and 1.55 arcseconds.

just the pushing force of $26612 \mathrm{~N}$ for each pin (design value). This value was calculated to compensate for the maximum forces expected during all the tests and phases of the launch considering the acceleration levels of $3.5 \mathrm{~g}$ lateral and $5 \mathrm{~g}$ axial.

According to Hertz theory the pressure at the contact area increases inversely with the diameter of the cavity and directly with the difference in diameter of the two surfaces in contact [32]:

$$
\sigma_{\max }=\frac{1.5 P^{1 / 3}}{0.721^{2} \pi C_{E}^{2 / 3}}\left(\frac{d_{2}-d_{1}}{d_{1} d_{2}}\right)^{2 / 3},
$$

where $\sigma_{\max }$ is the maximum stress in the contact area, $P$ is the load, $d_{1}$ and $d_{2}$ are the diameters of the pin and the spherical cavity, $C_{E}=\left[\left(1-v_{1}^{2}\right) / E_{1}\right]+\left[\left(1-v_{2}^{2}\right) / E_{2}\right]$, and $E_{1}, E_{2}$ and $v_{1}, v_{2}$ are the Young moduli and the Poisson ratios for the pin and the spherical cavity, respectively. With the constraint to maintain the stress at the contact area below the admissible value of $545 \mathrm{MPa}$, the minimization of the cavity size implied the use of very strict radius tolerances: $17 \mathrm{~mm}+0 /-0.10$ for the cavity and $16.8+0 /-0.04$ for the pin. In the worst combination of tolerances, that is, with the maximum radius of the cavity
TABLE 3: Analytical and numerical contact pressure in separation system interface.

\begin{tabular}{lccc}
\hline & $\begin{array}{c}\text { Nominal } \\
\text { geometry }\end{array}$ & $\begin{array}{c}\text { Worst tolerance } \\
\text { condition }\end{array}$ & $\begin{array}{c}\text { Admissible } \\
\text { stress }\end{array}$ \\
\hline Hertz formula & $371.05 \mathrm{MPa}$ & $419.17 \mathrm{MPa}$ & $545 \mathrm{MPa}$ \\
FEA & $423 \mathrm{MPa}$ & $455 \mathrm{MPa}$ & $545 \mathrm{MPa}$ \\
\hline
\end{tabular}

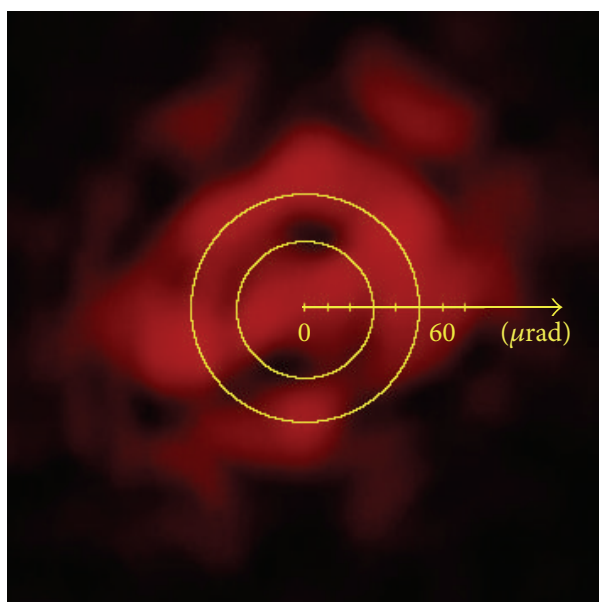

FIGURE 9: FFDP of CCR mounted in breadboard at $150^{\circ} \mathrm{C}$ and exposed to shroud at $-190^{\circ} \mathrm{C}$.

$(17 \mathrm{~mm})$ and the minimum pin diameter $16.76 \mathrm{~mm}$, one obtains, using both the analytical Hertz formula and Finite Element Analysis (FEA), the results reported in Table 3. The goodness of those results was confirmed by compression tests performed on a breadboard perfectly replicating the separation system interface with verification of absence of permanent deformations in the cavity.

In conclusion LARES is an almost perfect test particle because of (i) its very high mass-to-surface ratio which is the highest with respect to any artificial orbiting body in the solar system, (ii) its single piece design, and (iii) its special separation system interface with no protruding parts and minimum size of hemispherical cavities. In Table 2 the main characteristics of LARES in comparison to the other laser 


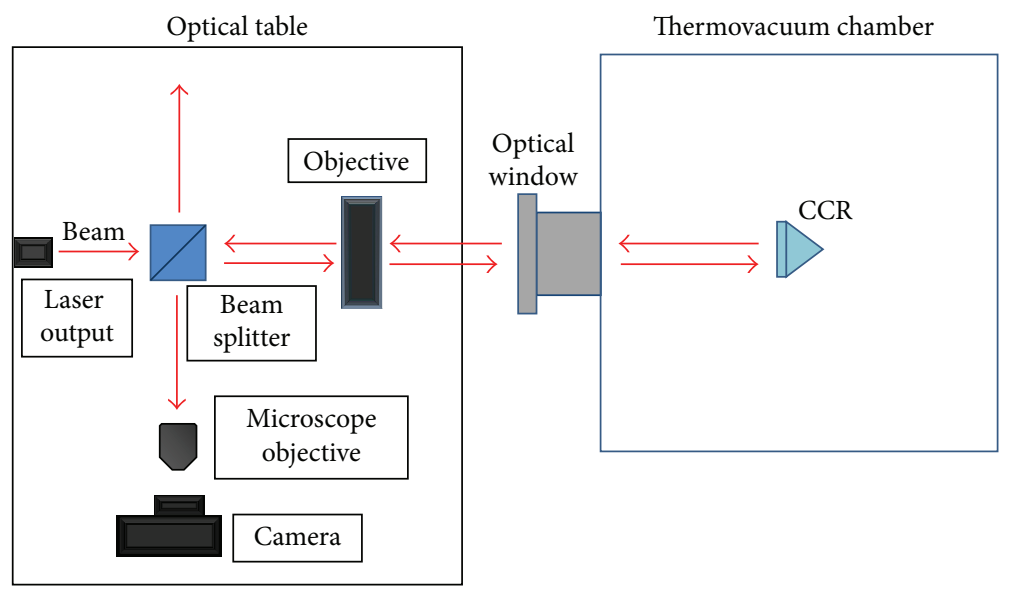

FIGURE 10: Schematic view of thermovacuum test setup.

ranged satellites mentioned in this paper are summarized. In particular one can see that the mass-to-surface ratio of LARES is 2.6 times bigger than the best one available since 1976 after the launch of LAGEOS.

\section{LARES Satellite Thermovacuum Tests}

Besides the classical environmental tests, typically performed on any space structure such as vibration and separation tests [33], for LARES, an extensive thermooptical campaign was required. Previous experience on retroreflectors issues allowed dealing with this specific problem within the short time available during the development of the project. The satellite body temperature could be estimated based on the measured values of emissivity $\varepsilon$ and absorptivity $\alpha$ of the tungsten alloy used [34]. With the experimental values of $\varepsilon$ and $\alpha$ it was possible with numerical simulations to determine that the satellite temperature in the worst operational case would have been about $140^{\circ} \mathrm{C}$. That in turn would have caused a temperature difference between the surface of the CCR and its apex (Figure 7) higher than the safety value of $\Delta T=2^{\circ} \mathrm{C}$.

Such high gradients would have deformed the dihedral angle of the CCR behind the safety value. Concern arose about the possibility to lose the return laser signal. The first solution proposed was to reduce the temperature of the satellite by passive thermal control using a surface treatment such as painting. Unfortunately this solution was not exempt from risks because the limited time before the launch could have not allowed appropriate long duration environmental tests for the coating. Furthermore the optical property of the coating or painting could change in time quickly and sensibly thus making the estimate of solar radiation pressure perturbation very difficult. It was therefore decided to verify directly and experimentally the optical behavior of the CCR in simulated operative conditions $[35,36]$. To this purpose a CCR inserted in a tungsten alloy cavity, Figure 7(b), perfectly replicating the ones used on the satellite, and controlled in temperature was subjected to the thermal loads expected in orbit. The specimen, suspended inside a thermovacuum chamber, was pointed towards the sun simulator, deep space (simulated with 5 nitrogen cooled walls), and Earth simulator (a black body disc maintained at $-20^{\circ} \mathrm{C}$ ) in different tests. After few hours of exposure in each test, once the equilibrium condition is reached, the CCR was pointed quickly, towards the optical circuit for acquiring an experimental Far Field Diffraction Pattern (FFDP). The FFDP is the pattern of energy distribution of the reflected laser pulse expected on the ground. In Figure 8 the FFDP of CCR n.51 is reported. It can be seen that there is negligible energy at the center. That is an imposed requirement so that the energy is well distributed in an annulus that is from $30 \mu \mathrm{rad}$ to $50 \mu \mathrm{rad}$ away from the center. That was possible because, during CCR manufacturing, the dihedral angles between the CCR back faces were increased by an amount between 1 and 2 arcseconds. This was a complex procedure that increased sensibly the cost of each CCR. The thermovacuum tests proved that there is enough energy in the annulus between $30 \mu \mathrm{rad}$ and $50 \mu \mathrm{rad}$ (Figure 9), which corresponds to a horizontal distance from the ground station of about $43.5 \mathrm{~m}$ and $72.5 \mathrm{~m}$, respectively (considering the satellite at $1450 \mathrm{~km}$ from the station). This deviation is due to the relative motion between the satellite and the station and to the speed of light that takes about $0.01 \mathrm{~s}$ for the round trip. In Figure 10 the setup of the thermocvacuum test is reported. An intensive experimentation was conducted on three different CCRs in all different conditions and hundreds of FFDPs have been acquired and analyzed. The results have demonstrated that the energy in the annulus changed maintaining enough energy in the annulus in each one of the test conditions, thus no surface treatment, besides cleaning, was required for the satellite.

\section{Conclusions}

The paper has described the scientific objectives and the engineering challenges of LARES mission. In particular the difficulties encountered during the manufacturing of the tungsten alloy were overcome by different techniques. Also the validity of the very critical thermooptical tests has been confirmed by the quality of the return signals so far acquired. 
Fifty stations of the ILRS are currently tracking LARES providing to the LARES team a reduction of the ranging data called normal points. The orbital determination analysis has confirmed that LARES is the best known test particle in the solar system. The final goal of reaching an accuracy of approximately $1 \%$ of the Lense-Thirring effect is expected in few more years because of the presence of periodical perturbations that need to be averaged out.

\section{Conflict of Interests}

The authors declare that there is no conflict of interests regarding the publication of this paper.

\section{Acknowledgments}

The authors gratefully acknowledge the support of the Italian Space Agency, Grants I/043/08/0 and I/043/08/1, and the International Laser Ranging Service for providing highquality laser ranging tracking of the LARES satellite. The authors wish to thank also the European Space Agency for having provided the qualification launch of VEGA.

\section{References}

[1] C. O. Alley, R. F. Chang, D. G. Currie et al., "Laser ranging retro-reflector: continuing measurements and expected results," Science, vol. 167, no. 3918, pp. 458-460, 1970.

[2] T. W. Murphy Jr., E. G. Adelberger, J. B. R. Battat et al., "Longterm degradation of optical devices on the moon," Icarus, vol. 208, no. 1, pp. 31-35, 2010.

[3] K. Sośnica, C. Baumann, D. Thaller, A. Jäggi, and R. Dach, "Combined LARES-LAGEOS solutions," in Proceedings of the 18th International Workshop on Laser Ranging, Fujiyoshida, Japan, November 2013.

[4] I. Ciufolini, D. Lucchesi, F. Vespe, and F. Chieppa, "Measurement of gravitomagnetism," EPL (Europhysics Letters), vol. 39, no. 4, pp. 359-364, 1997.

[5] I. Clutolini and E. C. Pavils, "A confirmation of the general relativistic prediction of the Lens-Thirring effect," Nature, vol. 431, no. 7011, pp. 958-960, 2004.

[6] I. Ciufolini, E. C. Pavlis, J. Ries et al., "Gravitomagnetism and its measurement with laser ranging to the LAGEOS satellites and GRACE earth gravity models," in General Relativity and John Archibald Wheeler, I. Ciufolini and R. A. Matzner, Eds., vol. 367 of Astrophysics and Space Science Library, pp. 371-434, Springer Science+Business Media B.V., Houten, The Netherlands, 2010.

[7] I. Ciufolini, A. Paolozzi, E. C. Pavlis et al., "Testing gravitational physics with satellite laser ranging," The European Physical Journal Plus, vol. 126, article 72, 2011.

[8] E. C. Pavlis, "The contribution of LARES in future ITRF developments," in Proceedings of the 2nd International LARES Science Workshop, Accademia dei Lincei, Roma, Italy, September 2012, http://www.lares-mission.com/ILSW2012/talks_2012/ ILSW2012_Pavlis.pdf.

[9] A. Einstein, Erklärung der Perihelbewegung des Merkur aus der allgemeinen [Relativitätstheorie Explanation of the Perihelion Motion of Mercury from the General Theory of Relativity], Sitzungsberichte der Preussischen Akademie der Wissenschaften, 1915.
[10] F. W. Dyson, A. S. Eddington, and C. Davidson, "A determination of the deflection of light by the sun's gravitational field, from observations made at the total eclipse of May 29, 1919," Philosophical Transactions of the Royal Society A: Mathematical, Physical and Engineering Sciences, vol. 220, no. 571-581, pp. 291333, 1920.

[11] J. P. Blaser, "Remarks by Heinrich Hertz (1857-94) on the equivalence principle," Classical Quantum Gravity, vol. 18, no. 13, pp. 2393-2395, 2001.

[12] S. Baeßler, B. R. Heckel, E. G. Adelberger, J. H. Gundlach, U. Schmidt, and H. E. Swanson, "Improved test of the equivalence principle for gravitational self-energy," Physical Review Letters, vol. 83, no. 18, pp. 3585-3588, 1999.

[13] R. F. C. Vessot and M. W. Levine, "A test of the equivalence principle using a space-borne clock," General Relativity and Gravitation, vol. 10, no. 3, pp. 181-204, 1979.

[14] R. F. C. Vessot, M. W. Levine, E. M. Mattison et al., "Test of relativistic gravitation with a space-borne hydrogen maser," Physical Review Letters, vol. 45, no. 26, pp. 2081-2084, 1980.

[15] J. H Taylor and J. M. Weisberg, "A new test of general relativitygravitational radiation and the binary pulsar PSR 1913+16," Astrophysical Journal, vol. 253, pp. 908-920, 1982.

[16] A. Einstein, Letter to Ernst Mach. Zurich, 25 June 1913, reported at pag. 544 of. C. W. Misner, K. S. Thorne, and J. A. Wheeler, Gravitation Freeman, San Francisco, Calif, USA, 1973.

[17] I. Ciufolini and F. Ricci, "Time delay due to spin inside a rotating shell," Classical and Quantum Gravity, vol. 19, no. 15, pp. 38753881, 2002.

[18] C. W. F. Everitt, D. B. Debra, B. W. Parkinson et al., "Gravity probe B: final results of a space experiment to test general relativity," Physical Review Letters, vol. 106, no. 22, Article ID 221101, 2011.

[19] J. Lense and H. Thirring, "On the influence of the proper rotation of central bodies on the motions of planets and moons according to Einstein's theory of gravitation," Physikalische Zeitschrift, vol. 19, pp. 156-163, 1918.

[20] I. Ciufolini, "Generalized geodesic deviation equation," Physical Review D, vol. 34, no. 4, pp. 1014-1017, 1986.

[21] W. M. Kaula, Theory of Satellite Geodesy, Blaisdell, Waltham, Mass, USA, 1966.

[22] I. Ciufolini, A. Paolozzi, and C. Paris, "Overview of the LARES mission: orbit, error analysis and technological aspects," Journal of Physics: Conference Series, vol. 354, no. 1, Article ID 012002, 2012.

[23] J. C. Ries, I. Ciufolini, E. C. Pavlis et al., "The Earth's framedragging via Laser-Ranged Satellites: a response to 'some considerations on the present-day results for the detection of frame-dragging after the final outcome of GP-B' by Iorio L.", European Physics Letters, vol. 96, no. 3, Article ID 30002, 2011.

[24] M. R. Pearlman, J. J. Degnan, and J. M. Bosworth, "The international laser ranging service," Advances in Space Research, vol. 30, no. 2, pp. 135-143, 2002.

[25] I. Ciufolini, A. Paolozzi, C. Paris, and G. Sindoni, "The LARES satellite and its minimization of the thermal forces," in Proceedings of the Metrology for Aerospace (MetroAeroSpace '14), pp. 299-303, IEEE, Benevento, Italy, May 2014.

[26] A. Paolozzi and I. Ciufolini, "LARES successfully launched in orbit: satellite and mission description," Acta Astronautica, vol. 91, pp. 313-321, 2013.

[27] A. Paolozzi, I. Ciufolini, E. Flamini et al., "Lares in orbit: some aspects of the mission," in Proceedings of the 63rd International 
Astronautical Congress (IAC '12), pp. 4468-4474, Naples, Italy, October 2012.

[28] A. Paolozzi, I. Ciufolini, and C. Vendittozzi, "Engineering and scientific aspects of LARES satellite," Acta Astronautica, vol. 69, no. 3-4, pp. 127-134, 2011.

[29] A. Bosco, C. Cantone, S. Dell'Agnello et al., "Probing gravity in NEO's with high-accuracy laser-ranged test masses," International Journal of Modern Physics D, vol. 16, no. 12a, pp. 22712285, 2007.

[30] I. Ciufolini, A. Paolozzi, E. C. Pavlis et al., "Testing general relativity and gravitational physics using the LARES satellite," The European Physical Journal Plus, vol. 127, article 133, 7 pages, 2012.

[31] R. M. German, P. Suri, and S. J. Park, "Review: liquid phase sintering," Journal of Materials Science, vol. 44, no. 1, pp. 1-39, 2009.

[32] W. C. Young and R. Budynas, Roark's Formulas for Stress and Strain, McGraw-Hill Professional, New York, NY, USA, 2011.

[33] A. Paolozzi, I. Ciufolini, G. Caputo et al., "LARES satellite and separation system," in Proceedings of the 63rd International Astronautical Congress (IAC '12), Paper: IAC-12-C2.9.5.x15247, Naples, Italy, October 2012.

[34] A. Paolozzi, I. Ciufolini, C. Vendittozzi, and F. Felli, "Material and surface properties of lares satellite," in Proceedings of the 63rd International Astronautical Congress (IAC '12), pp. 65596565, Naples, Italy, October 2012.

[35] A. Paolozzi, I. Ciufolini, C. Paris, G. Battaglia, and N. Reinhart, "Thermal tests on lares satellite components," in Proceedings of the 63rd International Astronautical Congress (IAC '12), pp. 6653-6657, Naples, Italy, October 2012.

[36] A. Paolozzi, I. Ciufolini, C. Paris, G. Sindoni, and D. Spano, "Qualification tests on the optical retro-reflectors of lares satellite," in Proceedings of the 63rd International Astronautical Congress (IAC '12), pp. 6280-6286, Naples, Italy, October 2012. 

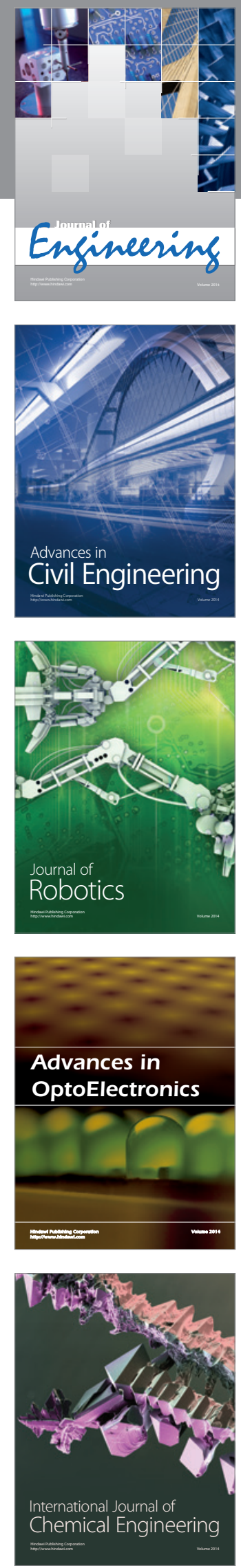

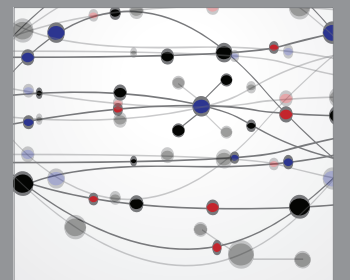

The Scientific World Journal
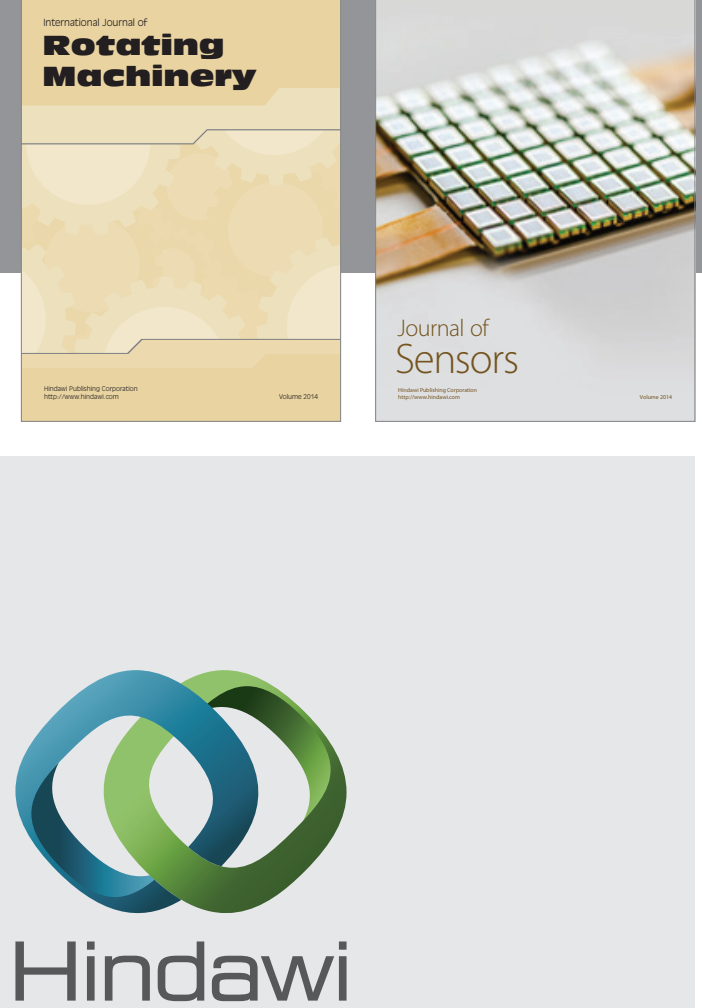

Submit your manuscripts at http://www.hindawi.com
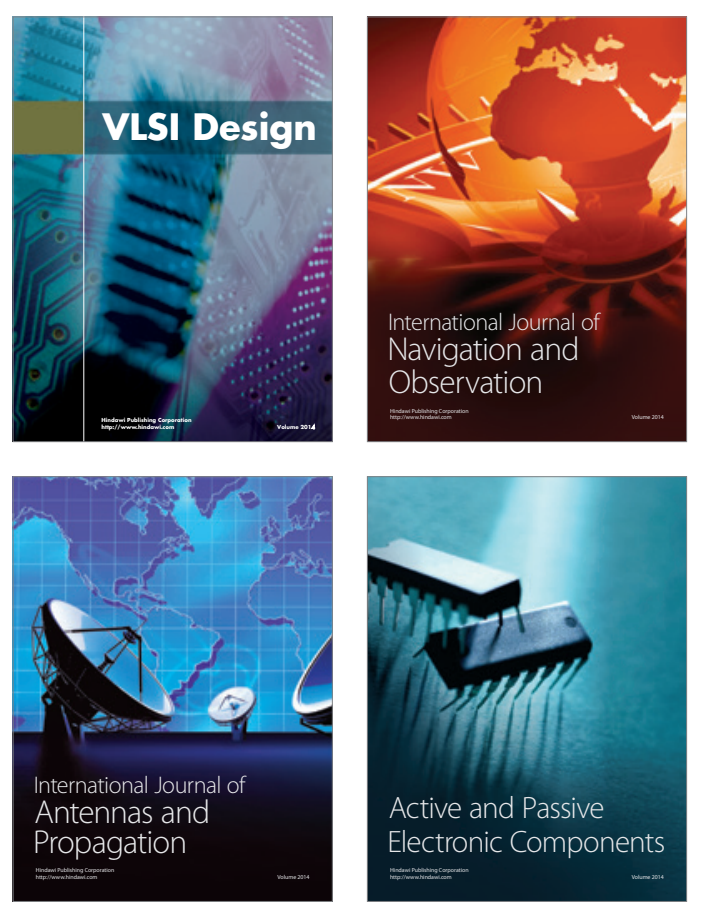
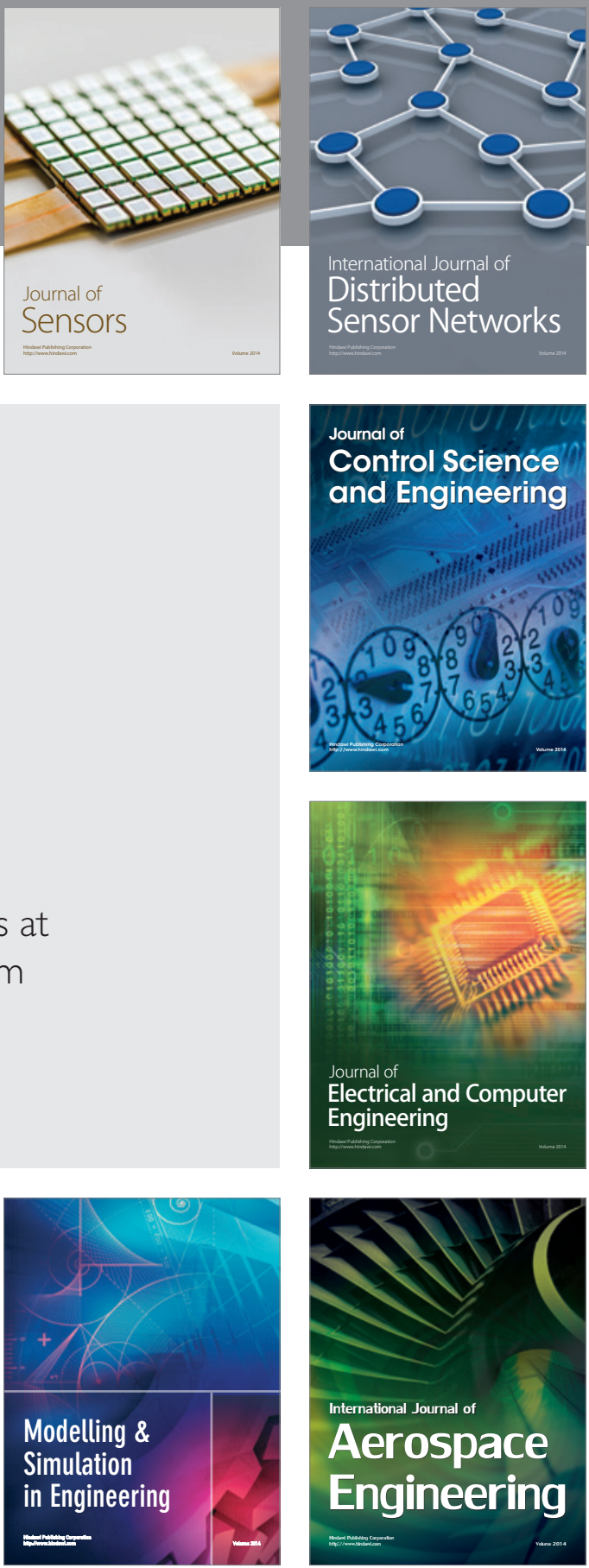

Journal of

Control Science

and Engineering
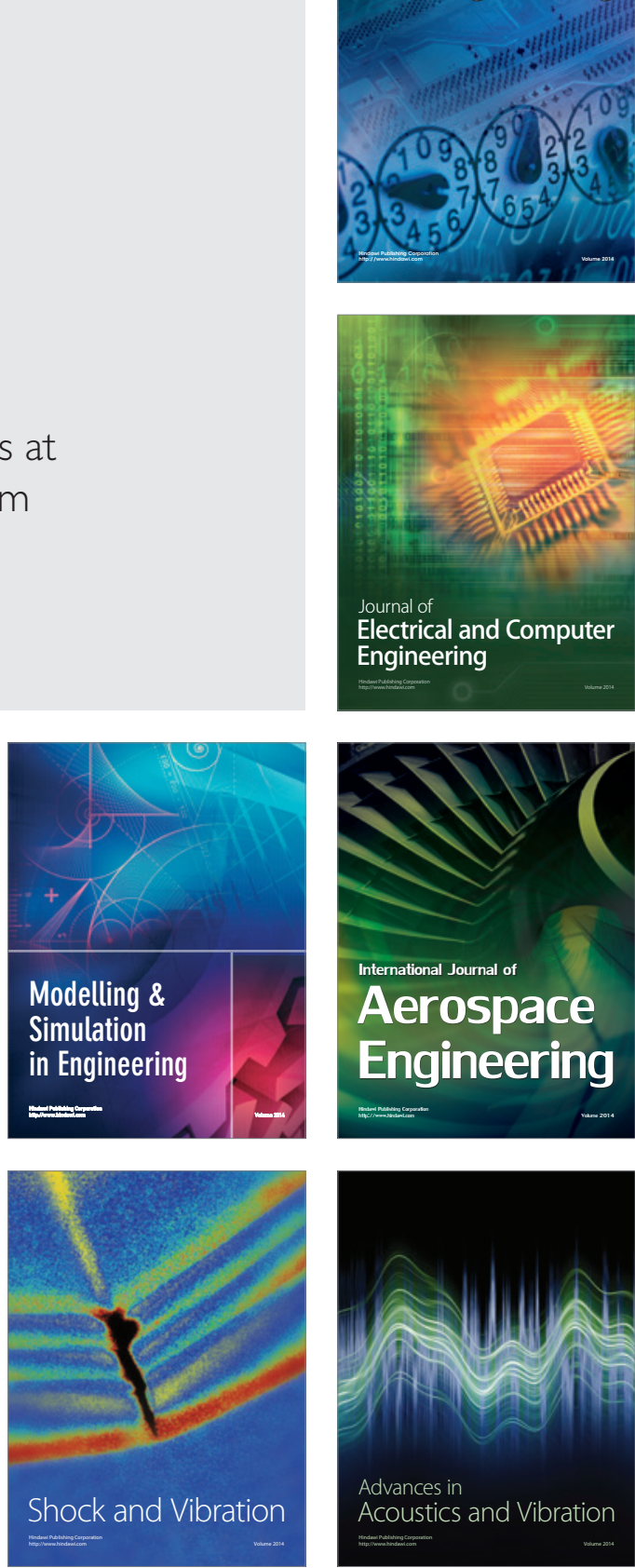